\title{
Indicadores de saúde e qualidade dos dados: uma análise do sistema de informação sobre nascidos vivos no Paraná, Brasil (1996 - 2018)
}

\author{
Health indicators and data quality: an analysis of the $e$ \\ information system on live births (sinasc) in Paraná, Brazil \\ (1996-2018)
}

Aline Veroneze de Mello, Zilda Pereira da Silva

Como citar este artigo:

MELLO, ALINE V.; SILVA, ZILDA

P.; Indicadores de saúde e

qualidade dos dados: uma análise do sistema de informação sobre nascidos vivos no Paraná, Brasil (1996 - 2018). Revista Saúde (Sta. Maria). 2021; 47 ( I)

\section{Autor correspondente: \\ Nome: Aline Veroneze de Mello \\ E-mail: alinemello@usp.br \\ Telefone: ( I I) 4272-0580 \\ Formação Profissional: Mestra em Ciências pelo Programa de Nutrição em Saúde Pública da Universidade de São Paulo (USP) São Paulo, SP, Brasil.}

Filiação Institucional: Faculdade de Saúde Pública, Universidade de São Paulo (USP)

Endereço para correspondencia:

Rua: Av. Dr. Arnaldo, 715 ,

Bairro: Cerqueira Cesar

Cidade: São Paulo

Estado: São Paulo

CEP: 01246904

Data de Submissão:

12/12/2020

Data de aceite:

| 4/06/202 |

Conflito de Interesse: Não há conflito de interesse

\section{(cc) $\mathrm{BY}-\mathrm{NC}-\mathrm{ND}$}

\section{RESUMO}

Objetivo: Analisar indicadores de saúde e a qualidade dos dados do SINASC no Paraná, Brasil, no período entre 1996 e 2018. Métodos: Foram analisados os dados do SINASC, disponibilizados pelo Ministério da Saúde em plataforma online (TabNet) nos anos de 1996, 2000, 2006, 2012 e 2018 no estado do Paraná dos principais indicadores de saúde, além de cobertura, completitude e consistência. Resultados: Quanto aos indicadores, houve um expressivo aumento na proporção de mães que realizaram 7 ou mais consultas pré-natal (1996: 49,38\% para 2018: 85,2\%), assim como houve aumento na proporção de partos do tipo cesárea, crianças nascidas pré-termo (1996: 4,25\% e 2018: 10,47\%) e com baixo peso (1996: 7,3\% e 2018: 8,5\%). Quanto a cobertura, o número de nascidos vivos obtidos pelo SINASC superior ao do Registro Civil (percentuais acima de 100\%). Contatou-se que para todas as variáveis a incompletitude dos dados foi excelente $(<5 \%)$. Para a consistência, a razão entre sexo masculino e feminino esteve próximo ou igual a 1,06 em todos os anos, peso ao nascer (associada ao período de duração da gestação), apresentou percentuais na categoria "correto" acima de 95\%. Considerações finais: Ressalta-se a importância de realizar o monitoramento das informações dos sistemas de estatísticas vitais no Paraná, além de indicadores de qualidade, uma vez que são fundamentais para nortear o preenchimento adequado dos dados, verificação de possíveis inconsistências, acompanhamento da situação de saúde da população, além de nortear políticas públicas focadas na saúde materno-infantil.

PALAVRAS-CHAVE: Indicadores; Saúde Materno-Infantil; Sistemas de Informação em Saúde; Nascidos Vivos.

\section{ABSTRACT}

Objective: The aim of the study was to analyze health indicators and the quality of SINASC data in Paraná, Brazil, from 1996 to 2018. Methods: The data of SINASC, made available by the Brazil Ministry of Health in an online platform (TabNet) in the years 1996, 2000, 2006, 2012 and 2018 in the state of Paraná were analyzed, in addition to coverage, completeness and consistency. Results: Regarding the indicators, there was a significant increase in the proportion of mothers who had 7 or more prenatal consultations (1996: $49.38 \%$ for $2018: 85.2 \%$ ), as well as an increase in the proportion of cesarean sections, children born preterm (1996: 4.25\% and 2018: 10.47\%) and underweight (1996: $7.3 \%$ and 2018: 8.5\%). Regarding coverage, the number of live births obtained by SINASC is higher than that of the Civil Registry (percentages above 100\%). It was reported that for all variables the incompleteness of data was excellent $(<5 \%)$. As for consistency, the ratio between male and female sex was close to or equal to 1.06 in all years, weight at birth (associated with the duration of pregnancy), presented percentages in the "correct" category above 95\%. Final considerations: Thus, the importance of monitoring information from vital statistics systems in Paraná is highlighted, as well as quality indicators, since they are fundamental to guide the proper filling out of data, verification of possible inconsistencies, monitoring of the health situation of the population, in addition to guiding public policies focused on maternal-infant health.

KEYWORDS: Health Information Systems; Indicators; Live Birth; Maternal and Child Health. 


\section{INTRODUÇÃO}

O Sistema de Informações sobre Nascidos Vivos foi desenvolvido pelo Sistema Único de Saúde (DATASUS), em 1990, no intuito de agrupar as informações epidemiológicas acerca dos nascimentos no Brasil1'. Essa análise pode ser feita a nível federal, por Unidades da Federação (UF), à nível municipal e regional por meio do sistema online TabNet (na seção estatística vitais - Nascidos vivos: 1996 a 2018) ou por meio da geração de arquivos em bancos de dados (TabWin)1. É importante ressaltar que o sistema permite acompanhar evoluções das estatísticas vitais ao longo do tempo (desde 1996) e, quando convertido em estudos, permite a proposição de ações e políticas públicas relativas à garantia, manutenção e/ou recuperação da saúde da mulher e infantil1.

Para a alimentação dos dados do SINASC é necessário o preenchimento das Declarações de Nascidos Vivos (DN), que são realizadas por cartórios (registro civil - nos últimos anos apenas informações relativas aos partos domiciliares), serviços de saúde, hospitais e maternidades². As Secretarias de Saúde, por sua vez, coletam as DN nos estabelecimentos de saúde e nos cartórios e atualizam as informações do sistema. O SINASC coleta aproximadamente 30 variáveis das $\mathrm{DN}^{2}$.

Neste sentido, o SINASC se torna uma ferramenta útil para análise de indicadores de saúde e qualidade das informações e, também, essencial à gestão, avaliação da situação de saúde e gerenciamento dos dados dos sistemas de informação em saúde, principalmente em termos de confiabilidade, validade, cobertura e completitude ${ }^{3,4}$. Ressalta-se que na avaliação das dimensões de qualidade os estudos frequentemente monitoram dados dos estados Rio de Janeiro e São Paulo4.

Mais especificamente no estado do Paraná, observam-se poucos estudos nacionais que avaliam indicadores e qualidade das estatísticas/variáveis relacionadas aos nascidos vivos ${ }^{5-7}$. Entre 1974 e 1994, estudo constatou uma melhora de cobertura das estatísticas vitais no estado, por meio do Registro Civil, devido ao aumento de partos hospitalares e diminuição no atraso de registros ${ }^{7}$. Outro estudo, que analisou características e indicadores dos nascidos vivos e de suas mães, residentes nos 22 municípios-sede de regionais de saúde do Paraná, em 2006, ainda que tenha identificado bons indicadores, mostrou que existem municípios com desigualdades sociais e que ainda precisam de recursos e políticas públicas específicas 5 .

Tendo em vista o exposto e a escassez de estudos que avaliam especificamente o estado do Paraná e a necessidade de conhecer as informações para tomadas de decisão, além de contribuir para a melhoria da qualidade das informações dos estados, o estudo tem por objetivo analisar indicadores de saúde e a qualidade dos dados do SINASC no Paraná, Brasil, no período entre 1996 e 2018. 


\section{MÉTODO}

Foram analisados os dados do SINASC, disponibilizados pelo Ministério da Saúde em plataforma online (TabNet) no período que compreendeu os anos de 1996, 2000, 2006, 2012 e 2018 no estado do Paraná, Brasil. Os dados foram extraídos da plataforma entre os meses de setembro e novembro de 2020. Com relação à análise de qualidade dos dados, foram utilizados como parâmetros: cobertura, completitude e consistência. Também foram avaliados indicadores de saúde ao longo dos anos. Os dados foram tabulados e calculados no Microsoft Excelß versão 2016.

\section{Indicadores de saúde}

Foi realizada uma análise dos principais indicadores de saúde: proporção de nascidos vivos de baixo peso (com peso inferior à 2500g), proporção de mães adolescentes (com idades entre 10 e 19 anos), proporção de nascidos vivos pré-termo (anterior a 37 semanas gestacionais), proporção de mães que realizaram 7 ou mais consultas pré-natal e a proporção de partos do tipo cesárea ${ }^{8}$. Foram calculadas proporções para os indicadores de saúde, utilizando a seguinte fórmula4:

\section{$\frac{\text { Número da variável de interesse }}{\text { Número denascidosvivosno ano }} \times 100$}

\section{Cobertura}

O número de nascidos vivos nos anos de 1996, 2000, 2006, 2012 e 2018 do SINASC foi comparado com as informações provenientes do Instituto Brasileiro de Geografia e Estatística (IBGE) que correspondem aos registros civis do estado do Paraná para os anos respectivos. Foi calculada a razão SINASC/IBGE em percentual3 .

\section{Completitude}

A completitude se refere aos dados completos de cada uma das variáveis analisadas. Os valores nulos, brancos ou ignorados (a porção de ausência das informações) são medidas utilizadas para identificar a qualidade dos dados (denominada "incompletitude"), sendo classificados em: excelente <5\%; bom, de 5 a 10\%; regular, de 10 a 20\%; ruim, de 20 a 50\%; e muito ruim, $>50 \%{ }^{6}$. Foram avaliadas quanto à incompletitude dos dados as seguintes variáveis do SINASC: sociodemográficas maternas (idade, escolaridade e estado civil), gravidez/parto (número de consultas pré-natal, duração da gestação, tipo de gravidez, tipo de parto e local de nascimento) e neonatais (sexo, cor/raça, peso ao nascer e anomalia congênita).

\section{Consistência}

Foi avaliada a consistência das variáveis neonatais sexo e peso ao nascer. A qualidade da variável sexo foi 
avaliada pelo cálculo da razão entre nascidos vivos do sexo masculino entre os nascidos vivos do sexo feminino no SINASC. Considerou-se como estabilidade biológica o valor próximo de 1,06. 0 peso ao nascer foi combinado com a variável duração da gestação e classificado entre três diferentes parâmetros que mensuram a qualidade da informação, sendo estes: errado, quando o peso ao nascer for inferior a 500g; correto, quando está entre os percentis 10 e 90 da curva de Lubchenco ${ }^{9}$ e provável erro, quando a gestação for superior a 37 semanas com o peso ao nascer da criança entre $1500 \mathrm{~g}$ e $2499 \mathrm{~g}$.

\section{RESULTADO}

Foram avaliados dados dos anos de 1996, 2000, 2006, 2012 e 2018. Quanto aos indicadores de saúde, observase que de 1996 a 2018 houve um expressivo aumento na proporção de mães que realizaram 7 ou mais consultas prénatal (1996: 49,38\%; 2000: 58,8\%; 2006: 74,8; 2012: 77,6\% e 2018: 85,2\%), assim como houve aumento na proporção de partos do tipo cesárea, crianças nascidas pré-termo (1996: 4,25\% e 2018: 10,47\%) e com baixo peso (com pouca variação - 1996: 7,3\% e 2018: 8,5\%) (Figura 1). Por outro lado, observou-se uma redução na proporção de mães adolescentes no estado (Figura 1).

Figura 1 - Proporção de indicadores de saúde relacionados à gravidez/parto, demográfico e neonatal, segundo ano. Paraná, Brasil (1996 - 2018).

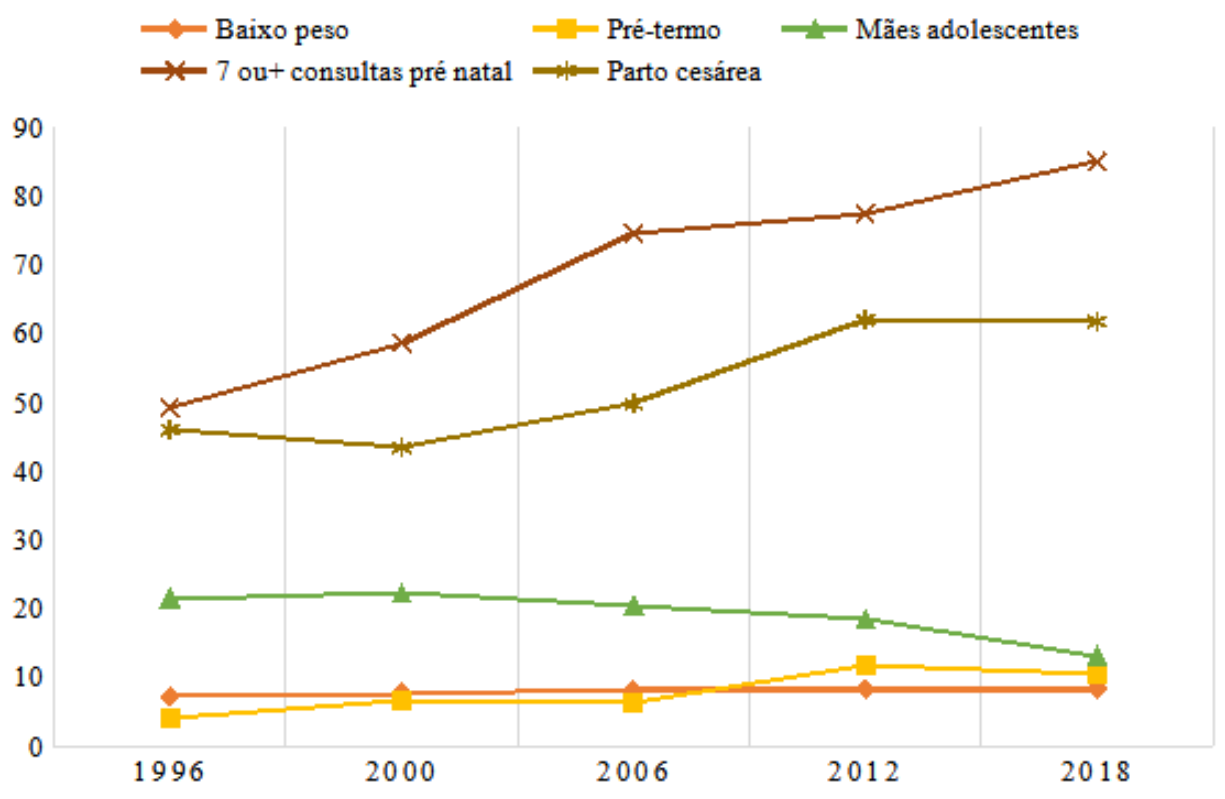

Fonte: SINASC: Sistema de Informação de Nascidos Vivos (1996, 200, 2006, 2012 e 2018);

Baixo peso: <2500g;

Nascidos pré-termo: anterior a 37 semanas gestacionais;

Mães adolescentes: com idades entre 10 e 19 anos. 
Com relação ao total de Nascidos vivos no Paraná, as informações do SINASC foram comparadas com os dados provenientes de estatísticas de Registro Civil disponibilizadas pelo IBGE para verificar a qualidade quanto a cobertura, que indicou em todos os anos avaliados, o número de nascidos vivos obtidos pelo SINASC superior ao do Registro Civil, demonstrada na razão SINASC/IBGE por valores percentuais acima de 100\%. A diferença passa a ser menor a partir de 2006 (Tabela 1).

Tabela 1 - Número e razão de nascidos vivos segundo fonte de informação e ano, conforme local de residência da mãe. Paraná, Brasil (1996 - 2018).

\begin{tabular}{cccc}
\hline Ano & SINASC & IBGE $^{*}$ & \% Razão (SINASC/IBGE) \\
\hline $\mathbf{1 9 9 6}$ & 196429 & 166532 & 117,95 \\
$\mathbf{2 0 0 0}$ & 179462 & 164321 & 109,21 \\
$\mathbf{2 0 0 6}$ & 153598 & 152553 & 100,69 \\
$\mathbf{2 0 1 2}$ & 153945 & 150445 & 102,33 \\
$\mathbf{2 0 1 8}$ & 156201 & 156141 & 100,04 \\
\hline
\end{tabular}

Fonte: SINASC: Sistema de Informação de Nascidos Vivos (1996, 2000, 2006, 2012, 2018);

IBGE: Instituto Brasileiro de Geografia e Estatística - Dados provenientes das estatísticas de registro civil (1996, 2000, 2006, 2012 e 2018).

No que se refere a qualidade dos dados, contatou-se que para todas as variáveis a incompletitude dos dados foi excelente (<5\%) no Paraná, a exceção do ano de 1996 em que a variável número de consultas pré-natal apresentou classificação "regular" (14,6\% de incompletitude). Variáveis que apresentaram classificação "NA" (não se aplica: estado civil, cor/raça e anomalia congênita), em 1996, ainda não constavam no formulário ou não foram coletadas informações de nascidos vivos e, em 2000, para variável anomalia congênita, as possíveis respostas "não e ignorado" estavam reunidas na mesma categoria, o que não possibilitou sua diferenciação (Tabela 2). 
Tabela 2 - Incompletitude das variáveis sociodemográficas, gravidez/parto e neonatais do Sistema de Informação de Nascidos Vivos (SINASC) do estado do Paraná, Brasil (1996 - 2018).

\begin{tabular}{|c|c|c|c|c|c|c|c|c|c|c|c|c|c|c|c|}
\hline \multirow[t]{2}{*}{ Variáveis } & \multicolumn{3}{|c|}{$\begin{array}{c}1996 \\
(n=196429)\end{array}$} & \multicolumn{3}{|c|}{$\begin{array}{c}2000 \\
(n=179462)\end{array}$} & \multicolumn{3}{|c|}{$\begin{array}{c}2006 \\
(n=153598)\end{array}$} & \multicolumn{3}{|c|}{$\begin{array}{c}2012 \\
(n=153945)\end{array}$} & \multicolumn{3}{|c|}{$\begin{array}{c}2018 \\
(n=156201)\end{array}$} \\
\hline & $\mathrm{n}$ & $\%$ & Categoria* & $\mathrm{n}$ & $\%$ & Categoria* & $\mathrm{n}$ & $\%$ & Categoria* & $\mathrm{n}$ & $\%$ & Categoria* & $\mathrm{n}$ & $\%$ & Categoria* \\
\hline \multicolumn{16}{|l|}{$\begin{array}{l}\text { Sociodemográficas } \\
\text { maternas }\end{array}$} \\
\hline Idade & 3270 & 1,66 & $E$ & 275 & 0,15 & $E$ & 4 & 0,00 & $E$ & 0 & 0,00 & $E$ & 0 & 0,00 & $E$ \\
\hline Escolaridade & 6327 & 3,22 & $E$ & 5207 & 2,90 & $E$ & 352 & 0,23 & $E$ & 355 & 0,23 & $E$ & 535 & 0,34 & $E$ \\
\hline Estado Civil & 196429 & 100 & $N A^{* *}$ & 1346 & 0,75 & $E$ & 1652 & 1,08 & $E$ & 631 & 0,41 & $E$ & 781 & 0,50 & $E$ \\
\hline $\begin{array}{l}\text { Gravidez/parto } \\
\text { Número de }\end{array}$ & & & & & & & & & & & & & & & \\
\hline $\begin{array}{l}\text { consultas pré-natal } \\
\text { Duração da }\end{array}$ & 28605 & 14,6 & $\mathrm{R}$ & 2539 & 1,41 & $E$ & 562 & 0,37 & $E$ & 1279 & 0,83 & $E$ & 138 & 0,09 & $E$ \\
\hline gestação & 2735 & 1,39 & $E$ & 696 & 0,39 & $E$ & 131 & 0,09 & $\mathrm{E}$ & 3283 & 2,13 & $\mathrm{E}$ & 818 & 0,52 & $\mathrm{E}$ \\
\hline Tipo de gravidez & 1919 & 0,98 & $E$ & 120 & 0,07 & $E$ & 47 & 0,03 & $E$ & 92 & 0,06 & $E$ & 121 & 0,08 & $E$ \\
\hline $\begin{array}{l}\text { Tipo de parto } \\
\text { Local de }\end{array}$ & 1582 & 0,81 & $\mathrm{E}$ & 276 & 0,15 & $\mathrm{E}$ & 121 & 0,08 & $\mathrm{E}$ & 83 & 0,05 & $\mathrm{E}$ & 86 & 0,06 & $\mathrm{E}$ \\
\hline $\begin{array}{l}\text { nascimento } \\
\text { Neonatais }\end{array}$ & 2315 & 1,18 & $E$ & 2 & 0,00 & $E$ & 11 & 0,01 & $E$ & 121 & 0,08 & $E$ & 4 & 0,00 & $E$ \\
\hline Sexo & 359 & 0,18 & $E$ & 49 & 0,03 & $E$ & 9 & 0,01 & $E$ & 11 & 0,01 & $\mathrm{E}$ & 12 & 0,01 & $E$ \\
\hline Cor/raça & 196424 & 100 & $N A^{* *}$ & 4300 & 2,40 & $E$ & 89 & 0,06 & E & 719 & 0,47 & $\mathrm{E}$ & 1500 & 0,96 & E \\
\hline Peso ao nascer & 1964 & 1,00 & $E$ & 647 & 0,36 & $\mathrm{E}$ & 55 & 0,04 & $E$ & 12 & 0,01 & $E$ & 3 & 0,00 & $E$ \\
\hline Anomalia congênita & 196424 & 100 & $N A^{* *}$ & 178450 & 99,33 & $N A^{* * *}$ & 252 & 0,16 & $E$ & 1129 & 0,73 & $E$ & 468 & 0,30 & $E$ \\
\hline
\end{tabular}

E: Excelente; B: Bom; R: Regular, NA: Não se aplica;

Fonte: SINASC: Sistema de Informações de Nascidos Vivos (1996, 2000, 2006, 2012 e 2018). Paraná, Brasil;

*Silva et al. 2013: excelente $<5 \%$; B, de 5 a $10 \%$; regular, de 10 a $20 \%$; ruim, de 20 a $50 \%$; e muito ruim, $>50 \%$;

**Variáveis não disponíveis/coletadas em 1996;

${ }^{* * *}$ Não e ignorado na mesma categoria. 
Ainda quanto a qualidade, avaliou-se a confiabilidade/consistência das variáveis neonatais. Para variável sexo, a razão entre sexo masculino e feminino esteve próximo ou igual a 1,06 em todos os anos, indicando uma boa estabilidade biológica e, portanto, trata-se de uma variável consistente. A variável peso ao nascer (associada ao período de duração da gestação), apresentou percentuais na categoria "correto" acima de 95\%. Observa-se que o "provável erro" foi diminuindo ao longo dos anos (1996: 4,9\%; 2000: 4,0\%; 2006: 3,9\%; 2012: 3,2\% e 2018: 2,9\%) e quanto à categoria "errado", os valores não chegam a 0,5\%, o que indica qualidade das informações (Tabela 3).

Tabela 3 - Avaliação da confiabilidade das variáveis neonatais (sexo e peso ao nascer) no SINASC. Paraná, Brasil (1996 - 2018).

\begin{tabular}{ccccccc}
\hline & \multicolumn{2}{c}{ Sexo* $^{*}$} & \multicolumn{3}{c}{ Peso ao nascer** } \\
Ano & $\begin{array}{c}\text { Masculino } \\
(\mathbf{n})\end{array}$ & $\begin{array}{c}\text { Feminino } \\
(\mathbf{n})\end{array}$ & $\begin{array}{c}\text { Razão } \\
\text { (Masculino/Feminino) }\end{array}$ & $\begin{array}{c}\text { Errado } \\
(\%)\end{array}$ & $\begin{array}{c}\text { Correto } \\
(\%)\end{array}$ & $\begin{array}{c}\text { Provável } \\
\text { erro }(\%)\end{array}$ \\
\hline $\mathbf{1 9 9 6}$ & 100479 & 95591 & 1,05 & 0,02 & 95,12 & 4,86 \\
$\mathbf{2 0 0 0}$ & 91535 & 87878 & 1,04 & 0,01 & 95,99 & 4,00 \\
$\mathbf{2 0 0 6}$ & 78201 & 75388 & 1,04 & 0,06 & 96,02 & 3,92 \\
$\mathbf{2 0 1 2}$ & 78680 & 75254 & 1,05 & 0,11 & 96,71 & 3,18 \\
$\mathbf{2 0 1 8}$ & 80197 & 75992 & 1,06 & 0,09 & 97,00 & 2,91 \\
\hline
\end{tabular}

Fonte: SINASC: Sistema de Informação de Nascidos Vivos (1996, 200, 2006, 2012 e 2018);

*Sexo: estabilidade biológica próxima do valor de 1,06 na ausência de interferências sociais e contextuais;

**Peso ao nascer: errado, peso inferior a 500g; correto, entre os percentis 10 e 90 da curva de Lubchenco ${ }^{9}$; provável erro, gestação superior a 37 semanas com peso entre 1500 e $2499 \mathrm{~g}$.

\section{DISCUSSÃO}

Os resultados deste estudo demonstram que mesmo com uma redução no número de mães adolescentes e aumento na proporção de mulheres com 7 ou mais consultas pré-natal, ainda há uma preocupação devido aos indicadores de proporção de partos do tipo cesárea, crianças nascidas pré-termo e com baixo peso terem aumentado no Paraná entre os anos de 1996 e 2018. Também indicam uma boa qualidade do SINASC quanto a completitude, cobertura e consistência.

O presente estudo identificou um aumento ao longo dos anos de crianças nascidas pré-termo e com baixo peso, um fato que se torna alarmante ao estado, tendo em vista que são fatores de risco fortemente associados à mortalidade infantil. A literatura indica que nascidos de baixo peso apresentaram 4,9 vezes mais chance de morrer do que aqueles com peso igual ou superior a $2500 \mathrm{~g}^{10}$. Neste mesmo sentido, se encontra o aumento da proporção de partos do tipo cesáreo no Paraná. Estudos demonstram que tem sido recorrente esse tipo de parto e muitas vezes pode não ser a opção ideal ${ }^{5}$. Ao encontro dos achados no Paraná, o Brasil apresenta altos índices de cesáreas o que a torna a principal forma de parto no país ${ }^{11}$. 
Em contrapartida, resultados positivos indicam menor proporção de mães adolescentes que passou de cerca de 20\% em 1996 para 13,2\% em 2018. A gravidez na adolescência pode trazer um impacto negativo na vida destas mulheres, pois muitas abandonam os estudos, ainda não possuem renda própria e podem passar por problemas como depressão e falta de apoio da família ${ }^{12}$.

Houve também um aumento da proporção de 7 ou mais consultas pré-natal que passou de $58,8 \%$ em 2000 e atingiu 85,2\%, em 2018. Um fato que pode ter contribuído para o aumento da proporção de 7 ou mais consultas pré-natal foi a instituição da Rede Mãe Paranaense, a partir de 2012, que organizou a atenção à saúde e ações do pré-natal e puerpério, assim como o acompanhamento do crescimento e desenvolvimento das crianças, principalmente no primeiro ano de vida ${ }^{13}$. Entre as suas ações estão 0 acompanhamento de no mínimo 7 consultas no pré-natal, realização de exames, classificação de risco das gestantes e das crianças e a garantia do parto, o que pode explicar os achados do presente estudo ${ }^{13}$.

No que se refere a cobertura dos dados, observou-se uma subestimação dos nascidos vivos por parte dos dados de Registro Civil do IBGE quando comparados com o SINASC (diferença que variou de 0,04 a 13,9\%). É importante ressaltar que o Registro Civil tem o objetivo de realizar a contagem do número de registros de nascimentos, enquanto 0 SINASC caracterizar as condições da gestação, do parto e do nascimento, o que torna as diferenças encontradas como algo a ser reavaliado pelos Registros Civis no estado ${ }^{14}$. A falta de regularidade e homogeneidade das informações nos cartórios sempre foi um problema, principalmente, devido a sua contribuição em problemas na cobertura atrelada ao subregistro das informações, um dos motivos pelo qual o SINASC foi criado'. Sendo assim, a principal questão que pode explicar as diferenças observadas, é devido a própria finalidade de criação do SINASC, ou seja, esperar-se-ia que os números de nascidos vivos registrados no SINASC superassem os registrados pelo IBGE.

Em termos de completitude, pode-se identificar um excelente preenchimento dos dados nas diferentes variáveis analisadas, com valores de incompletitude inferiores a 5\%, a exceção do ano de 1996 em que a variável número de consultas pré-natal apresentou classificação "R" (de 10 a 20\% de incompletitude), portanto, indicando uma preocupação no preenchimento das declarações de nascidos vivos que vem permanecendo ao longo do tempo. Outros estudos demonstraram o mesmo resultado para o estado do Paraná, indicando excelente preenchimento ${ }^{6,15-16}$. Neste sentido, destaca-se a importância da completitude dos dados, como indicado, em estudo de revisão sistemática que avaliou os métodos para identificar completitude, principalmente, no sentido a se evitar problemas estruturais nos instrumentos de coleta de dados, dificuldades e falta de treinamento dos entrevistadores, bem como dos indivíduos responsáveis por atualizar o sistema de informação ${ }^{17}$.

Quanto a consistência das informações neonatais, as variáveis sexo (valores próximos a 1,06 em todos os anos avaliados) e peso ao nascer (mais de 95\% com informações corretas e redução das informações provavelmente 
incorretas ao longo dos anos) mostraram-se consistentes. Estudo que avaliou a qualidade da informação das variáveis do SINASC do ano 2002, quanto à consistência dos resultados obtidos (variável sexo) para todas as Unidades da Federação (UF) do Brasil, inclusive o Paraná, apresentou valores próximos a 1,065 .

Em relação ao peso ao nascer, estudo que utilizou o indicador de consistência de peso ao nascer no Brasil, mostrou que para categoria "correto", houve variação de 93,67\% (no estado do Maranhão) a 96,13\% (na Paraíba) e 0 Paraná apresentou 94,9\%. No Maranhão e na Paraíba, encontraram-se os maiores e menores percentuais de "provável erro": 6,16\% e 3,83\%, respectivamente. Já no Paraná, o "provável erro" foi de 5,01\%, o que se mostra semelhante ao presente estudo. Também é importante destacar que ao longo dos anos houve menores percentuais de "provável erro" e aumento do "correto", o que ratifica a melhora gradual da consistência e consequente qualidade das informações do SINASC, Paraná15.

\section{CONSIDERAÇÕES FINAIS}

Observou-se que entre os parâmetros de qualidade cobertura, completitude e consistência das variáveis de estudo do SINASC, no estado do Paraná, que já se apresentavam adequados e com boa qualidade, melhoraram ainda mais com o passar dos anos na comparação entre os anos de 1996, 2000, 2006, 2012 e 2018. No quesito indicadores, ainda que tenha reduzido a proporção de mães adolescentes e aumentado a proporção de mulheres com 7 ou mais consultas pré-natal, houve aumento na proporção de partos do tipo cesárea, crianças nascidas pré-termo e com baixo peso. Sendo assim, ressalta-se a importância de realizar o monitoramento das informações dos sistemas de estatísticas vitais no Paraná, além de indicadores de qualidade, uma vez que são fundamentais para nortear o preenchimento adequado dos dados, verificação de possíveis inconsistências, acompanhamento da situação de saúde da população, além de nortear políticas públicas focadas na saúde materno-infantil.

\section{REFERÊNCIAS}

1 - BRASIL. Ministério da Saúde. SINASC - Sistema de Informações de Nascidos Vivos. Brasília; 2020. [citado 2020 set. 28]. Disponível em: <http://www2.datasus.gov.br/DATASUS/index.php?area=060702>.

2 - BRASIL. Ministério da Saúde. Notas técnicas do Sistema de Informações sobre Nascidos Vivos; 2017. Brasília; 2017. [citado 2020 out. 01]. Disponível em: <http://tabnet.datasus.gov.br/cgi/sinasc//Nascidos_Vivos_1994_2012. pdf>. 
3 - RIPSA. Rede Interagencial de Informações para a Saúde. Indicadores básicos para a saúde no Brasil: conceitos e aplicações. OPAS: Brasília, 2008.

4 - Lima CRA, Schiramm JMA, Coeli, CM, Silva MEM. Revisão das dimensões de qualidade dos dados e métodos aplicados na avaliação dos sistemas de informação em saúde. Cadernos de Saúde Pública, Rio de Janeiro. 2009;25(10).

5 - Melo EC, Mathias TAF. Distribuição e autocorrelação espacial de indicadores da saúde da mulher e da criança, no Estado do Paraná, Brasil. Revista Latino-Americana de Enfermagem. 2010; 18(6), 1-10.

6 - Silva GF, Aidar T, Mathias TAF. Qualidade do Sistema de Informações de Nascidos Vivos no estado do Paraná, 2000 a 2005. Revista da Escola de Enfermagem da USP, 2011; 45(1), 79-86.

7 - Davanso SM, Ribas AMM. Avaliação das estatísticas de nascidos vivos no Paraná 1974-1994. Anais ABEP - X Encontro Nacional de Estudos populacionais, 2016; 247-261.

8 - BRASIL. Ministério da Saúde. A experiência brasileira em sistemas de informação em saúde. Organização Pan-Americana da Saúde, Fundação Oswaldo Cruz. Brasília: Editora do Ministério da Saúde, 2009 (Série B. Textos Básicos de Saúde).

9 - LUBCHENCO LO, HANSMAN C, DRESSLER M, BOUD E. Intrauterine growth as estimated from liveborn birth weight data at 24 to 42 weeks of gestation. Pediatrics 1963;32:793-800.

10 - Mombelli MA, Sass A, Molena CAF, Téston EF, Marcon SS. Fatores de risco para mortalidade infantil em municípios do Estado do Paraná, de 1997 a 2008. Revista Paulista de Pediatria, 2012; 30(2), 187-194.

11 - Rodrigues JCT, Almeida IESR, Neto AGO, Moreira TA. Cesariana no Brasil: uma análise epidemiológica. Revista Multitexto, 2016; 4(1), 48-53.

12 - Vieira AM, Santos DGS, Guimarães TMM. Fatores que interferem na assistência ao pré-natal de gestantes adolescentes. Research, Society and Development, 2020; 9(10), e1419108546-e1419108546. 
13 - SECRETARIA DA SAÚdE DO PARANÁ. Mãe Paranaense. Secretaria da Saúde. Estado do Paraná, 2019. [citado 2020 out. 27]. Disponível em: <https://www.saude.pr.gov.br/Pagina/Mae-Paranaense>.

14 - Szwarcwald CL, Leal MC, Esteves-Pereira AP, Almeida WS, Frias PG, Damacena GN et al. Avaliação das informações do Sistema de Informações sobre Nascidos Vivos (SINASC), Brasil. Cadernos de Saúde Pública, 2019; 35(10), e00214918, 2019.

15 - Romero DE, Cunha CB. Avaliação da qualidade das variáveis epidemiológicas e demográficas do Sistema de Informações sobre Nascidos Vivos, 2002. Cadernos de Saúde Pública, 2007; 23(3), 701-714.

16 - Oliveira MM, Andrade SSCA, Dimech GS, Oliveira JCG, Malta DC, Rabello N et al. Avaliação do sistema de informações sobre nascidos vivos. Brasil, 2006 a 2010. Epidemiologia e Serviços de Saúde, 2015; 24(4), 629-640.

17 - Correia LOS, Padilha BM, Vasconcelos SML. Métodos para avaliar a completitude dos dados dos sistemas de informação em saúde do Brasil: uma revisão sistemática. Ciência \& Saúde Coletiva, 2014; 19(11), 4467-4478. 